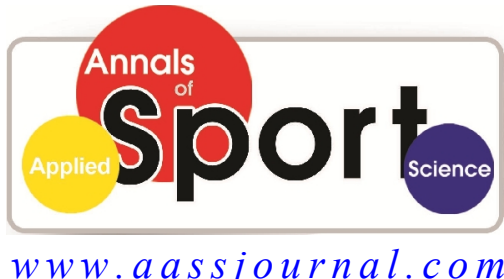

ISSN (Online): $2322-4479$

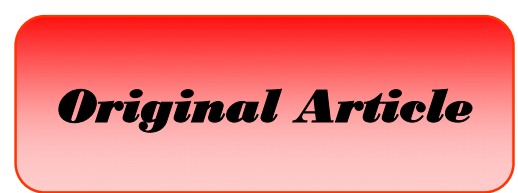

Received: $31 / 01 / 2014$

Accepted: 29/03/2014

\title{
Bowlers' Performances in 2013 Champions Trophy
}

\section{R. Indika P. Wickramasinghe*}

- Department of Mathematical Sciences, Eastern New Mexico University, Portales, NM 88130, USA.

\begin{abstract}
Cricket is becoming a fashionable team game throughout the world. One day internationals (ODI) have been able to bring more and more spectators to the cricket ground, due to the nature of its' format. Among the ODI tournaments, the Champions trophy takes a significant place, as it is the second largest tournament after the global event of cricket world cup. This paper investigates the performances of the bowlers who appeared in the 2013 Champions trophy. The main aim of this study is to identify the bowlers' characteristics which impact their performances. The kernel resampling technique (KRT) is implemented to analyze the collected sample $(\mathrm{N}=41)$ of the bowlers who played the Champions trophy in 2013. Findings of this study reveal that the bowlers' prior economy rate and the height have influenced the performance significantly, while the handedness and the bowling style have not shown a statistical significance on their performances.
\end{abstract}

Key Words: ODI Cricket, Bowler's Performances, Prior Economy Rate, Height, Handedness, Bowling Style.

Corresponding Author: 


\section{INTRODUCTION}

Cricket is a bat and ball team game played over fifty countries in the world in different levels. Though test cricket, ODI, and T20 are the three facets of the game of cricket, the attention in this paper is given to the ODI format. Batting, bowling, and fielding are the three main departments of the game. Among all the cricket related statistics, batting and bowling are the most popular statistics among cricket loving people. There was a high demand for cricket related studies with the popularity of the game. When considering ODI tournaments, the Champions trophy is a major tournament organized by the international cricket council (ICC), the governing body of cricket. The ICC Champions trophy was played approximately for every two years time, and the 2013 tournament is believed as the concluding event. This was appeared as an ICC Knock out competition in 1998 and they changed the name as Champions Trophy in 2002. Initially all the ICC cricket teams took part in this competition and after 2009 , only the first eight teams (according to the ICC ranking) got the opportunity to play in the tournament. Out of all the ICC teams, so far only South Africa, New Zealand, India, Sri Lanka, West Indies, and Australia have been able to win the Champions trophy.

In June 2013, the final Champions trophy was held in England and Wales with the participation of the eight teams. Australia (AUS), England (ENG), India (IND), New Zealand (NZ), Pakistan (PAK), Sri Lanka (SL), South Africa (SA), and West Indies (WI) were the teams who participated to the tournament. Based on their performances, India and England qualified to play the final match of the tournament. At the end, the Indian team was able to win the 2013 Champions trophy by beating the England team.
Both bowling and batting statistics are rich in the game of cricket, but the majority of the studies in the literature are discussing about the batting statistics. Therefore, the aim of this paper is to investigate the bowlers' performances in 2013 Champions trophy. Mukerjee (1) discussed a methodology to quantify bowlers' performances by applying the knowledge of Social Network Analysis (SNA). Dey et al. (2) investigated the performances of bowlers in the Indian Premier League (IPL) using a multiple criteria decision analysis (MCDA) methodology. Portus et al. (3) discussed the impact of bowling eight overs (bowling six balls) at a stretch (8-over spell) on the bowler's performance. Christie (4) focused on the physical demands of fast bowlers, and pointed out the necessity of physiological studies to study the performances of fast bowlers. Loram et al. (5) determined the fast bowlers' bowling technique by considering the bowlers' knee biomechanics. In their studies, Kimber (6) and Van Staden (7) proposed graphical methods to compare the performances of bowlers. Phillips (8) conducted a multidisciplinary cross-sectional study investigation about the talentdevelopment of fast bowlers. Hurrion (9) studied the fast-medium-bowlers' back-foot and front-foot strike at the delivery stride. Lemmer (10) showed how to adapt the bowling performance in ODI format into Twenty20. Thelwell and Maynard (11) studied about cricketers' performance indicators using a psychological approach. Sharma (12) utilized a factor analysis approach to analyze the performance of cricketers.

Literature reveals that the average runs per over, the number of wickets taken, and the strike rate are some of the accepted criteria used in quantifying bowlers' performances. Dey et al. (2) studied the bowlers' performances in the Indian Premier League (Indian professional league for 
cricketers of T20 format, IPL). In their investigation, authors have used six different measurements, namely the bowling economy rate, bowler's average, bowler's strike rate, the number of wickets taken, the total number of overs bowled, and the total number of matches played during one particular session of IPL. Sharp et al. (13) and Lemmer (14) used three bowlers' performance indicators, such as the economy rate, the average, and the strike rate to analyze bowlers' performances in their studies. Kimber (6) and Van Staden (7) utilized graphical methods to compare the performances of bowlers. Beaudoin and Swartz $(15,16)$ developed a statistic using the popular Duckworth-Lewis methodology to evaluate the bowlers' performances. In order to quantify bowling performance, Lemmer (17) constructed a statistic, the combined bowling rate, $\mathrm{CBR}$, by combining the traditional bowling measures, the average, the economy rate and the strike rate into a single measure. In Lemmer (18) he extended this measure for the case of a small number of overs bowled, by allocating weights to the wickets the bowler had taken. The measure was indicated by CBR as can be seen in equation 1 to follow. Basevi and Binoy (19) used some of the bowlers' statistics, such as the number of runs conceded, the number of wickets taken, and the number of balls bowled to quantify bowlers' performances. In their studies, Lewis (20) and Lemmer (10) discussed about the limitations of traditional performance indicators related to the assessment of player performance. Lewis (20) and Lemmer (10) argued to construct a novel statistic by combining the traditionally used bowler statistics to quantify the bowlers' performance.

\section{MATERIALS AND METHODS}

Subjects. In this study, 41 bowlers were selected using numerous criteria from all the eight teams. As the first criterion, bowlers who played more than two matches, and got at least a wicket in the tournament were considered. In addition, bowlers who have bowled at least 10 overs during the tournament were considered. Bowler related statistics, such as the number of overs bowled, the number of maiden overs (overs that did not concede runs), the number of runs scored against the bowler, and the number of wickets taken in each game were recorded in this study. Each bowler's career economy rate (The average runs scored in an over) prior to the tournament, handedness, height, and the bowling style also were recorded.

According to the table 1, the prior average economy rate of bowler was 4.87 $(\mathrm{SD}=0.57)$. During this tournament, the mean number of matches played by a bowler was $3.56(\mathrm{SD}=0.77)$, and the mean number of overs a bowler bowled was 24.35 $(\mathrm{SD}=9.00)$. The average number of wickets taken by a bowler was 4.37 ( $\mathrm{SD}=2.89)$. During this tournament, a bowler has given away an average of $119.44(\mathrm{SD}=47.353)$ runs and about $3.15(\mathrm{SD}=3.09)$ of extra runs (wide balls and no-balls) to the opposite team. As the table 1 shows, $61 \%(\mathrm{SD}=0.49)$ of the bowlers were not spinners and about $73 \%(\mathrm{SD}=0.46)$ of the bowlers were right handed. The average height of a bowler is 1.83 meters $(\mathrm{SD}=0.10)$. Bowler's bowling style was categorized as 0 (spinner) and 1 (non-spinner), while the handedness of the bowler was represented as 0 (left-handed) and 1 (right-handed). 
Table 1: Description of bowlers in the 2013Champions Trophy, $(\mathrm{N}=41)$

\begin{tabular}{cccc}
\hline Variable & Mean & SD & Coding \\
\hline Econ & 4.87 & 0.57 & \\
\hline Matches & 3.56 & 0.77 & \\
\hline Overs & 24.35 & 9.00 & \\
\hline Runs & 119.44 & 47.35 & \\
\hline Wickets & 4.37 & 2.89 & \\
\hline Extras & 3.15 & 3.09 & 0 -spinner, 1-non spinner \\
\hline Bowling_Style & 0.61 & 0.49 & 0-left, 1-right \\
\hline Hand & 0.73 & 0.45 & \\
\hline Height & 1.83 & 0.10 & \\
\hline
\end{tabular}

Instruments / Statistical Analysis. Lemmer (18) introduced a statistic called combined bowling rate (CBR) for quantifying bowlers' performances. This statistic is a function of various performance indications of bowlers, and calculated according to the following equation:

Equation (1): $\mathrm{CBR}=\frac{3 \mathrm{R}}{\left(\mathrm{V}^{*}+\mathrm{O}+\mathrm{W}^{*} \times \mathrm{R} / \mathrm{B}\right)}$

Here $\mathrm{R}$ represents the number of runs conceded by the bowler, $\mathrm{O}$ denotes the number of overs bowled, $B$ represents the number of balls bowled, and $\mathrm{W}^{*}$ is the sum of the weights of the wickets taken by the bowler. These weights were assigned for each wicket as explained by Lemmer (10).

As mentioned before, CBR summarizes most of the performance indicators that were discussed before. A better bowler is expected to have a smaller CBR value. Throughout this analysis, CBR is used as the measurement of quantifying the bowlers' performances. The accuracy of multiple regression analysis technique is problematic for a small sample $(\mathrm{N}=41)$ like this. As Bai and Pan (21) stated, there are several issues of using multiple regression when it is applied to a small data set. Bai and Pan (21) introduced an appealing and an accurate alternative technique called, kernel resamping technique (KRT) to replace the multiple regression for small samples.
Wickramasinghe (22) used KRT with a small sample of data to analyze the students' performances of statistics class. As Bai and Pan (21) explained, KRT can be summarized as follows.

Consider a multivariate set of small size data set, $X_{1}, X_{2}, \ldots ., X_{n}$ from a $d$-dimensional space $R^{d}$. Here, $d$ is the number of variables and $n$ is the size of the sample.

Step 1. A multivariate Gaussian kernel is defined:

$$
K_{i}(x) \sim N_{d}\left(X_{i}, H_{o}^{2}\right), i=1,2, \ldots, n
$$

Where:

$$
H_{o}=\left(\frac{4}{d+2}\right)^{\left(\frac{1}{d+4}\right)} \sum^{\frac{1}{2}} n^{\left(-\frac{1}{d+4}\right)}
$$

Here $\Sigma$ is the covariance matrix.

Step 2. Let $\mathrm{X}_{1}^{*}, \mathrm{X}_{2}^{*} \ldots \ldots . \mathrm{X}_{\mathrm{n}}^{*}$, a sample drawn from Gaussian kernel $\mathrm{K}_{\mathrm{i}}(\mathrm{x})$.

Step 3. Multiple regression is conducted over the above drawn sample.

Step 4. Steps 2 to 3 is repeated for $\mathrm{K}$ times (in this study $\mathrm{K}=300$ ).

Step 5. The sampling distributions of the results of the multiple regressions are evaluated. 
A Matlab program was written by the author to implement this procedure. In this analysis, CBR value is the response variable $(Y)$ and Bowling_Style $\left(X_{1}\right)$, Handedness $\left(X_{2}\right)$, Econ $\left(X_{3}\right)$, and the height $\left(X_{4}\right)$ are the explanatory variables. Therefore, our aim is to estimate the parameters as shown in the following regression model:

Equation(2): $Y_{i}=\beta_{0}+\beta_{1} X_{i 1}+\beta_{2} X_{i 2}+\beta_{3} X_{i 3}+$ $\beta_{4} X_{i 4}+\varepsilon_{i} ; i=1,2, \ldots, N$
Here the error term follows a normal distribution with zero mean and constant variance.

\section{RESULTS}

CBR values were calculated for each bowler in the sample $(\mathrm{N}=41)$ using the equation 1. Table 2 shows the 10 players according to the lowest CBR values. According to the table 1, RA Jadeja has recorded the most number of wickets (W) in the tournament, but KD Mills is the bowler with the smallest CBR value.

Table 2: First top 10 players according to the CBR rankings

\begin{tabular}{cccccc}
\hline Bowling & Country & O & R & W & CBR \\
\hline KD Mills & NZ & 16.3 & 63 & 6 & 6.87 \\
\hline RA Jadeja & IND & 41 & 154 & 12 & 6.91 \\
\hline R McLaren & SA & 17.1 & 78 & 5 & 7.02 \\
\hline JM Anderson & ENG & 37 & 151 & 11 & 7.59 \\
\hline D Vettori & NZ & 21 & 66 & 2 & 7.81 \\
\hline CJ McKay & AUS & 24 & 99 & 5 & 8.37 \\
\hline M Hafeez & PAK & 16.1 & 60 & 2 & 8.79 \\
\hline MJ McClenaghan & NZ & 23.5 & 144 & 11 & 8.84 \\
\hline M Irfan & PAK & 20 & 83 & 4 & 8.86 \\
\hline R Ashwin & IND & 41 & 181 & 8 & 9.02
\end{tabular}

According to the figure 1, Indian spinners have performed better than all the other spinners in this Champions trophy tournament though there is no statistical evidence. When the performances of nonspinners (fast bowlers and medium pacers) are considered, New Zealanders have performed better than the other bowlers. According to the figure 1, there is a performance gap between spinners and nonspinners in each of the country as far as the mean CBR value is concerned. The performance gap is less for both English and Sri Lankan teams in comparison with the other teams. Non-spinners from England, New Zealand and South Africa have performed better than their spinners while, Indian, Pakistan, Sri Lankan, and West Indian spinners have performed better than their non-spinners. Australian spinners were discarded in this analysis as they did not meet the selection criteria.

When investigating the impact of the handedness and the bowling style on player performance, as the figure 2 indicates the left handed spinners have performed better than the rest of the bowlers. Right handed non-spinners have performed slightly better than the left handed spinners. Among all the bowlers, the performances of left handed non-spinners are the lowest.

When analyzing the performances of bowlers, it is important to consider the number of extra runs that they have given away during the tournament. According to the figure 3, English spinners have conceded the minimum number of average extra runs while their non-spinners have conceded the highest average number of extras. Though most of the spinners from each country have 
given away less average number of extras Zealand bowlers have indicated the contrary. than their non-spinners, Indian and $\mathrm{New}$
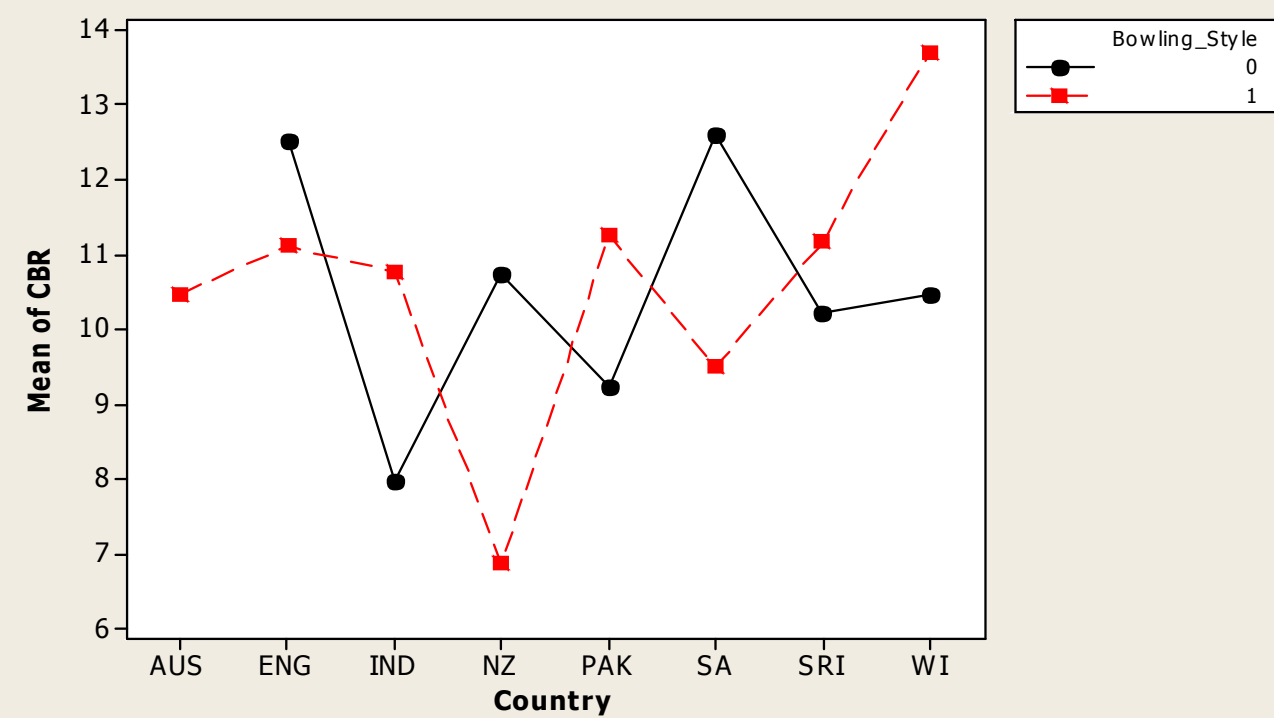

Figure 1: Performance of bowlers from each country. Bowling style (0-spinner, 1-nonspinner).

When answering the question of what bowler's characteristics influenced his performance during the Champions trophy, KRT method was implemented by setting the value of $K=300$. Table 3 shows the parameter estimation of parameters $\left(\hat{\beta}_{j}, \mathrm{j}=0\right.$, $1,2,3,4)$ in equation 2 using Kermel Resamping Technique (KRT), Ordinary Least-Squares (OLS), and Bootstrapping Regression (BR) methods. As T-values indicate, only the economy rate and the height have significantly influenced the player performance in the tournament irrespective of the three estimation methods. While the economy rate has positively influenced the CBR, the height of the bowler has negatively influenced $\mathrm{CBR}$ value. According to the coefficients of determination values, only the KRT method gives the highest value of 59.5. Other two methods, OLS and BR methods give smaller values of 33.50 and 22.55 respectively. As far as the standard errors of the estimators are concerned, KRT shows the smallest standard errors.

According to the figure 2, though there is slight evidence that the handedness impacts the bowlers' performances, table 3 shows that this influence is not statistically significant. Further, table 3 indicates that the bowlers' performances are not influenced by their bowling styles.

\section{DISCUSSION}

In this paper, bowlers' performances in the Champions trophy were investigated. As motioned before, there are several popular performance indicators that we often see in the literature. Bowler's economy rate, numbers of wickets, and bowler's strike rate to quantify the bowler's performance are some of the instances. According to the literature, Lewis (20) and Lemmer (10) pointed out that the previously mentioned 
statistics are unable to provide accurate results in evaluating the bowlers' performances. Therefore, a new statistic called CBR introduced by Lemmer (18) was considered in this study as the performance indicator. For improving the accuracy with small sample inference, KRT method was utilized as an alternative for multiple regression method. Using the comparison of the three estimation methods, namely KRT,
OLS, and BR, KRT outperformed the other two methods as far as the accuracy of the estimators is concerned. As Bai and Pan (21) pointed out, it is evident that the KRT method performed better than both OLS and BR for small sample multiple regression analysis. The outcomes of this study also proved that the KRT outperformed the other two methods.

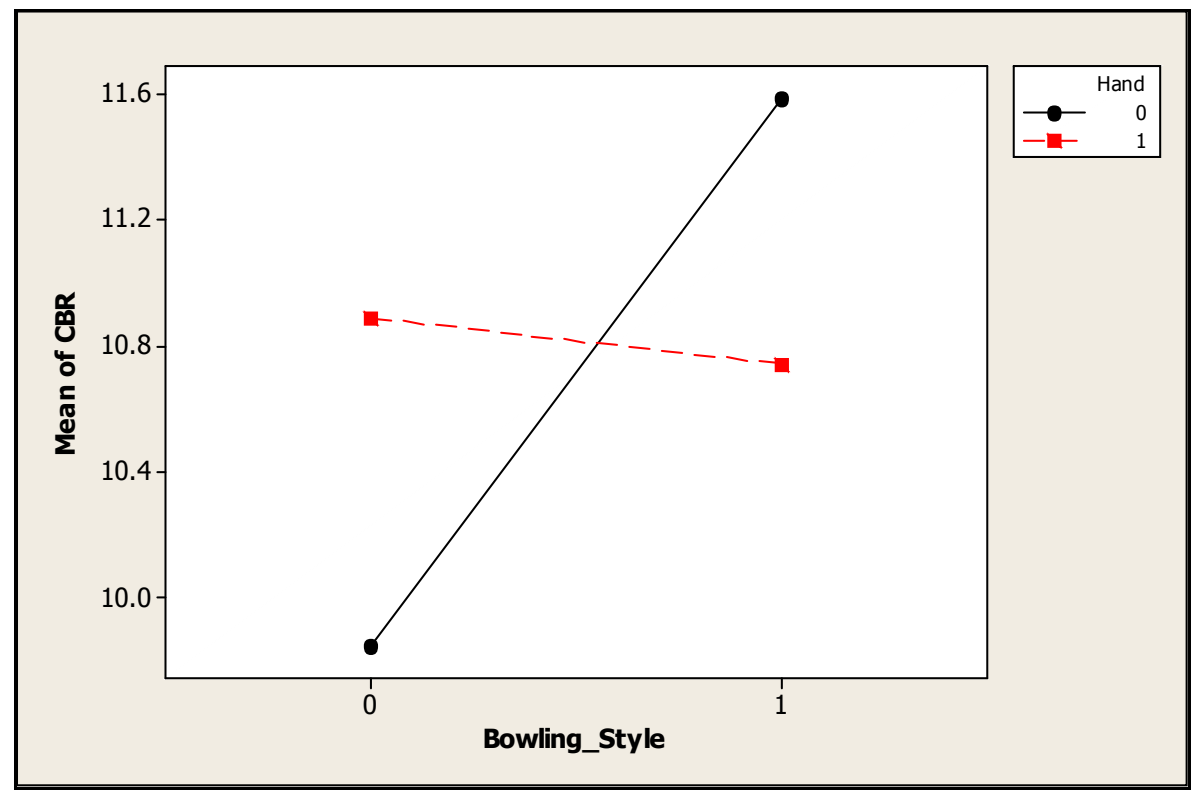

Figure 2: Handedness (0-left, 1-right), Bowling style (0-spinner, 1-non-spinner) and the Performance.

According to the findings, both bowler's prior economy rate and the height have significantly influenced player performance in the Champions trophy. This indicates that the bowler's prior economy rate is proportional to the $\mathrm{CBR}$ value. This makes sense as it is reasonable to expect a better performance from a bowler who has less economy rate than a bowler with a higher economy rate.

Table 3: Results of the KRT $(K=300)$, OLS, and BR $(B=300)$

\begin{tabular}{|c|c|c|c|c|c|c|c|c|c|}
\hline \multirow[b]{2}{*}{$\begin{array}{c}\text { Independent } \\
\text { Variable }\end{array}$} & \multicolumn{3}{|c|}{$\beta$} & \multicolumn{3}{|c|}{ SE ( $\beta$ ) } & \multicolumn{3}{|c|}{$\mathbf{T}$} \\
\hline & KRT & OLS & BR & KRT & OLS & BR & KRT & OLS & BR \\
\hline Constant & 29.12 & 31.47 & 35.83 & 7.60 & 8.16 & 9.08 & $3.83 *$ & $3.86 *$ & $5.11 *$ \\
\hline Bowling_Style & 0.86 & -0.99 & -1.71 & 0.78 & 0.85 & 0.97 & 1.10 & -1.32 & -1.62 \\
\hline Handedness & -1.11 & -1.21 & -2.19 & 0.80 & 0.88 & 0.93 & -1.38 & -1.50 & -1.98 \\
\hline Econ & 1.90 & 1.87 & 3.72 & 0.63 & 0.73 & 0.75 & $3.02 *$ & $3.02 *$ & $3.75^{*}$ \\
\hline Height & -14.94 & -15.08 & -21.57 & 4.25 & 4.35 & 5.07 & $-3.52 *$ & $-3.63 *$ & $-5.37^{*}$ \\
\hline
\end{tabular}




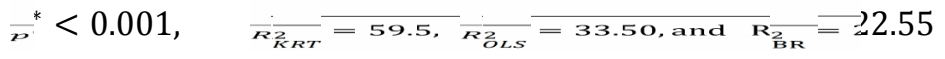

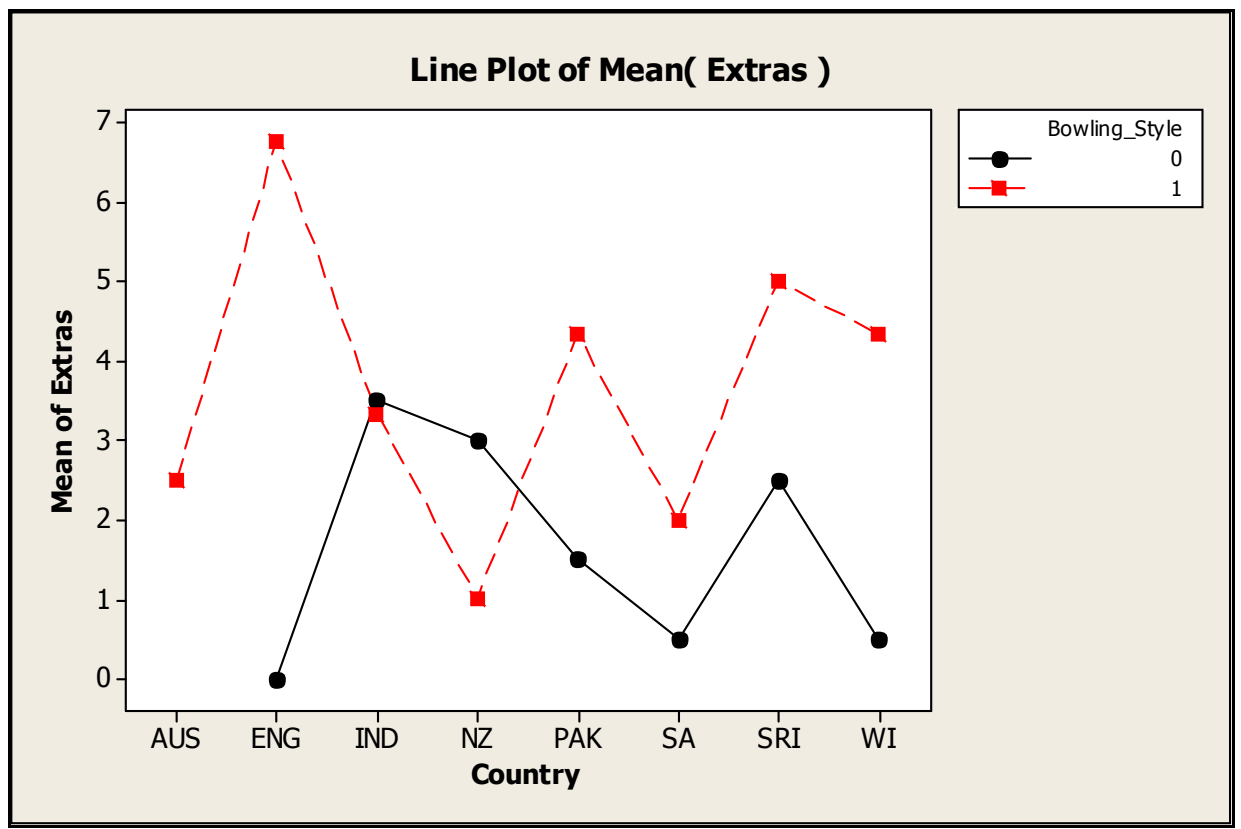

Figure 3: Mean number of extra runs conceded by bowlers of each country

According the table3, with the increment of bowler's height his CBR value decreases. This indicates that taller bowlers have got an advantage as far as their performance is concerned. This present outcome of the study goes to provide a justification to the findings of Pyne et al. (23). As the table 2 indicated, most of the bowlers who have reserved a top place in the CBR ranking are taller than 1.88 meters (except $\mathrm{M}$ Hafeez who is 1.74 meters tall). This may be due to the fact that a taller bowler can bring the cricket ball up in significant amount, after hitting the ball on the turf than the other bowlers. This can create a pressure on the batsman as he finds it difficult to score runs. Though the handedness and bowling style of the bowler do not demonstrate any statistical significance on their performances, it would be very interesting to investigate this issue in a major tournament like ICC world cup. Due to the small data set, it was difficult to consider other bowling styles, such as fast bowling, fast medium, medium fast, and medium into this analysis.

\section{REFFRENCES}

1. Mukherjee S. Quantifying individual performance in Cricket - A network analysis of batsmen and bowlers. Physica A: Statistical Mechanics and its Applications. 2014;393(0):624-37.

2. Dey PK, Ghosh DN, Mondal AC. A MCDM Approach for Evaluating Bowlers Performance in IPL. Journal of Emerging Trends in Computing and Information Sciences. 2011;2(11):563-73.

3. Portus MR, Sinclair PJ, Burke ST, Moore DJ, Farhart PJ. Cricket fast bowling performance and technique and the influence of selected physical factors during an 8-over spell. Journal of sports sciences. 2000;18(12):9991011. Epub 2001/01/04.

4. Christie CJ-A. The Physical Demands of Batting and Fast Bowling in Cricket. In: Zaslav KR, editor. An International Perspective on Topics in Sports Medicine and Sports Injury: InTech; 2012. p. 321-33. 
5. Loram LC, McKinon W, Wormgoor S, Rogers GG, Nowak I, Harden LM. Determinants of ball release speed in schoolboy fast-medium bowlers in cricket. The Journal of sports medicine and physical fitness. 2005;45(4):483 90. Epub 2006/02/01.

6. Kimber A. A Graphical Display for Comparing Bowlers in Cricket. Teaching Statistics. 1993;15(3):84-6.

7. Van Staden PJ. Comparison of cricketers' bowling and batting performances using graphical displays. Current Science. 2009;96(6):764-6.

8. Phillips EJ. A multidisciplinary examination of fast bowling talent development in cricket [PhD]: Queensland University of Technology; 2011.

9. Hurrion PD, Dyson R, Hale T. Simultaneous measurement of back and front foot ground reaction forces during the same delivery stride of the fast-medium bowler. Journal of sports sciences. 2000;18(12):993-7. Epub 2001/01/04.

10. Lemmer HH. An analysis of players' performances in the first cricket Twenty20 world cup series. South African Journal for Research in Sport, Physical Education and Recreation. 2008;30(2):71-7.

11. Thelwell RC, Maynard IW. The effects of a mental skills package on 'repeatable good performance' in cricketers. Psychology of Sport and Exercise. 2003;4(4):377-96.

12. Sharma SK. A Factor Analysis Approach in Performance Analysis of T-20 Cricket. Journal of Reliability and Statistical Studies. 2013;6(1):69-76.

13. Sharp GD, Brettenny WJ, Gonsalves JW, Lourens M, Stretch RA. Integer optimisation for the selection of a Twenty20 cricket team. Journal of the Operational Research Society. 2011;62(9):1688-94.

14. Lemmer HH. Team selection after a short cricket series. European Journal of Sport Science. 2011;13(2):200-6.

15. Beaudoin D. The Best Batsmen and Bowlers in One-Day Cricket. British Columbia, Canada: Simon Fraser University; 2003.

16. Beaudoin D, Swartz T. The best batsmen and bowlers in one-day cricket: general. South African Statistical Journal. 2003;37(2):203-22.

17. Lemmer HH. The combined bowling rate as a measure of bowling performance in cricket. South African Journal for Research in Sport, Physical Education and Recreation. 2002;24(2):37-44.

18. Lemmer HH. A method for the comparison of the bowling performances of bowlers in a match or a series of matches. South African Journal for Research in Sport, Physical Education and Recreation. 2005;27(1):75-87.

19. Basevi T, Binoy G. The world's best Twenty20 players. 2007; Available from: http://contentrsa.cricinfo.com/columns/content/story/311962.html.

20. Lewis AJ. Towards fairer measures of player performance in one-day cricket. Journal of the Operational Research Society. 2005;56(7):804-15.

21. Bai H, Pan W. An Application of a New Multivariate Resampling Method to Multiple Regression. Multiple Linear Regression Viewpoints. 2009;35:1-5.

22. Wickramasinghe RIP. Factors Influencing Students' Success in an Online Statistics Course at College-level. Journal of Mathematical Sciences \& Mathematics Education. 2014;9(1):27-34.

23. Pyne DB, Duthie GM, Saunders PU, Petersen CA, Portus MR. Anthropometric and strength correlates of fast bowling speed in junior and senior cricketers. Journal of strength and conditioning research / National Strength \& Conditioning Association. 2006;20(3):620-6. Epub 2006/08/30. 


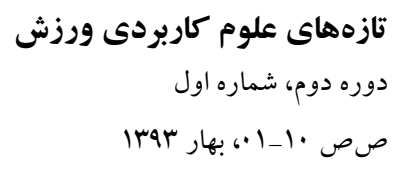

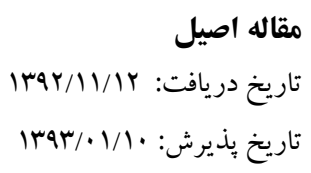

مقاله اصيل

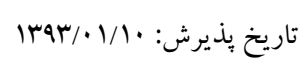

\section{بررسى اجراى ير تابكنندَان كريكت

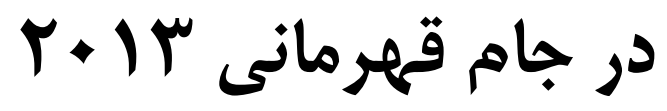 \\ آر. اينديكا بيى. ويكر اماسينغَ}

استاديار كروه علوم رياضى، دانشخاه نيومكزيكو شرقى، يورتالز، امريكا.

جكيده

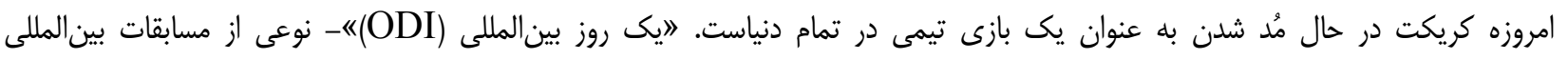

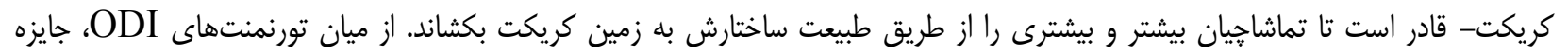

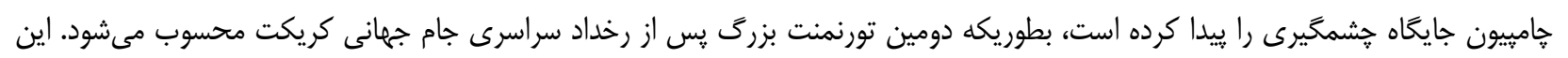

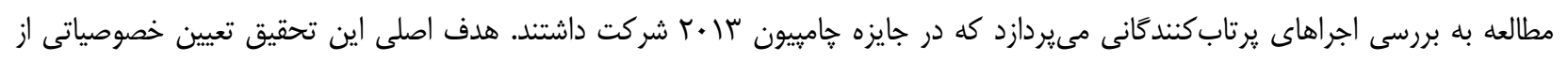

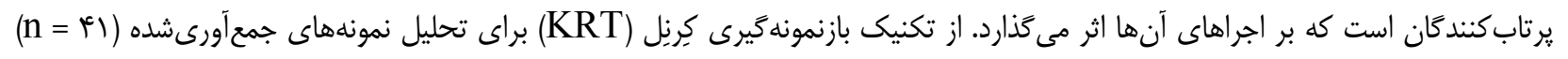

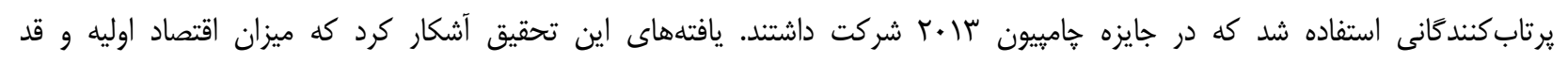

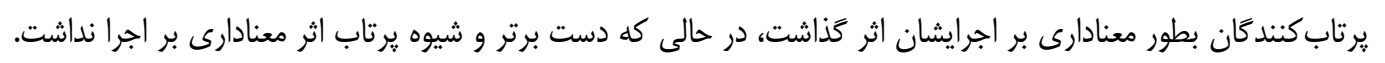

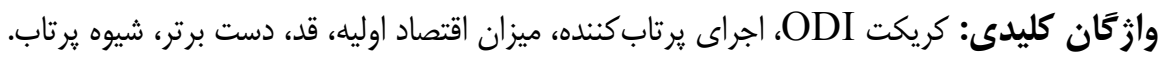

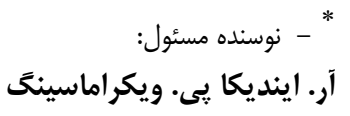

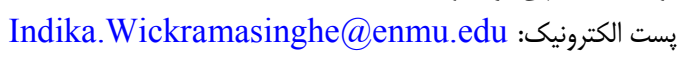

